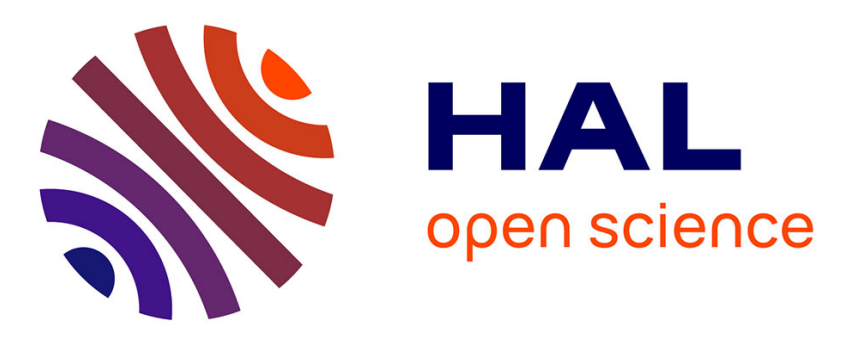

\title{
Interlaced Direct Adaptive Regulation Scheme Applied to a Benchmark Problem
}

\author{
Ioan Doré Landau, Abraham Castellanos Silva, Luc Dugard, Xu Chen
}

\section{To cite this version:}

Ioan Doré Landau, Abraham Castellanos Silva, Luc Dugard, Xu Chen. Interlaced Direct Adaptive Regulation Scheme Applied to a Benchmark Problem. MED 2016 - 24th Mediterranean Conference on Control and Automation, Mediterranean, Jun 2016, Athènes, Greece. pp.443-448, 10.1109/MED.2016.7535848 . hal-01314563

\section{HAL Id: hal-01314563 https://hal.science/hal-01314563}

Submitted on 11 May 2016

HAL is a multi-disciplinary open access archive for the deposit and dissemination of scientific research documents, whether they are published or not. The documents may come from teaching and research institutions in France or abroad, or from public or private research centers.
L'archive ouverte pluridisciplinaire HAL, est destinée au dépôt et à la diffusion de documents scientifiques de niveau recherche, publiés ou non, émanant des établissements d'enseignement et de recherche français ou étrangers, des laboratoires publics ou privés. 


\title{
Interlaced Direct Adaptive Regulation Scheme Applied to a Benchmark Problem
}

\author{
Ioan Doré Landau*,Abraham Castellanos Silva*, \\ Luc Dugard*, Xu Chen ${ }^{\dagger}$.
}

\begin{abstract}
Direct adaptive regulation schemes using Internal Model Principle and FIR Youla-Kučera controller parametrization have been extensively used for attenuation of multiple unknown and time varying narrow band disturbances [10], [5], [2].This approach provides very good results but requires a very careful design of the central controller in order to keep the water bed effect on the output sensitivity function at a an acceptable level. To simplify the design of the central controller, an adaptive regulation scheme is proposed in this paper which will incorporate a particular adaptive IIR YoulaKučera Filter, called $\rho$-notch structure (the denominator is a projection inside the unit circle of the poles of the model of the disturbance which has roots on the unit circle). The adaptive scheme estimates separately the numerator and denominator parameters of the IIR Youla-Kučera Filter. The use of this approach drastically simplify the design of the central controller and provides even better results than [2] with the advantage to use a single central controller independently of the number of narrow band disturbances. Real-time results obtained on an active vibration control system will illustrate the potential of this approach. Comparison with other approaches applied to the same system are also provided.
\end{abstract}

Index Terms-Adaptive Regulation, Active Vibration Control, Inertial Actuators, Multiple Narrow Band Disturbances, YoulaKučera Parametrization, Internal Model Principle

\section{INTRODUCTION}

The problem come from the fact that the Internal Model Principle does too much by assuring asymptotically total rejection of the disturbance while in practice attenuation of narrow band disturbances by 40 to $60 \mathrm{~dB}$ is largely enough.

A first approach for overcoming the problem induced by the Internal Model Principle has been considered in [1]. Instead of the IMP one uses Band Stop Filters (BSF) centered at the frequencies of the spikes which allow to introduce a finite attenuation. The implementation use an indirect adaptation scheme which time consuming.

The novel approach proposed in this paper is based on the use of an IIR Youla Kucera parametrization. The objective is to use the degree of freedom offered by the denominator of the IIR Youla Kucera filter in order to assign in real time additional poles to the closed loop which will allow to reduce the water bed effect and to improve robustness. To do this it was found convenient to to use the $\rho-$ notch filters of the form $D_{p}\left(z^{-} 1\right) / D_{p}\left(\rho z^{-} 1\right)$ with $0<\rho<1$ where $D_{p}\left(z^{-} 1\right)$ is the denominator of the model of the narrow band disturbance ( with roots on the unit circle). This approach will be comparatively evaluated with repsect to the best results obtained previously within the EJC Benchmark on adaptive regulation [6]

\section{Plant DESCRIPTION AND PROBLEM STATEMENT}

A photo of the active vibration control experimental set up used in this study is presented in fig. 1 along with the basic actions performed by the system. A detailed description can be found in [6].

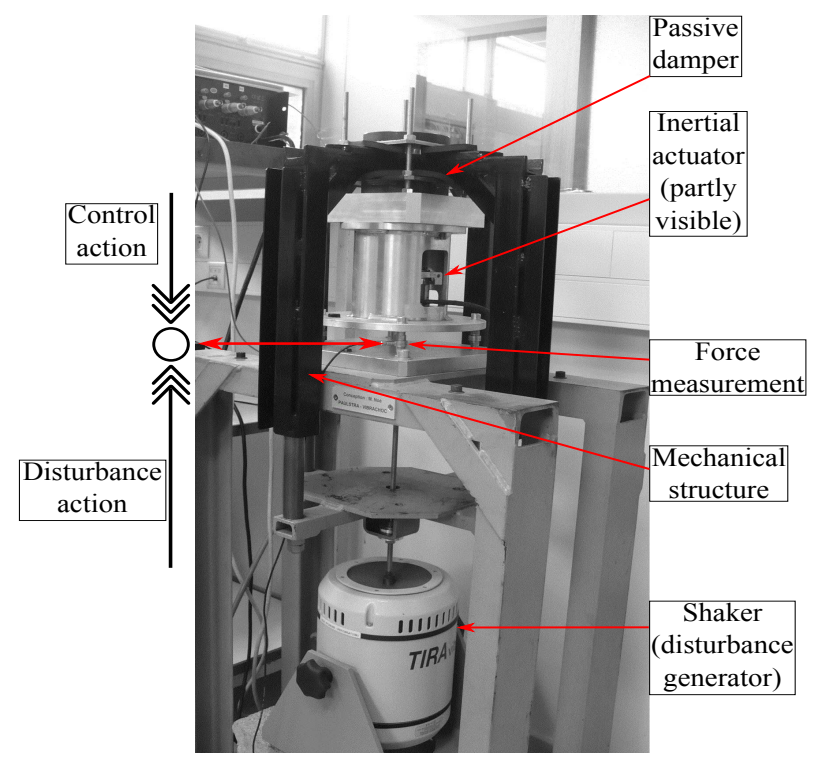

Fig. 1. Active vibration control using an inertial actuator (photo).

The system consists of a passive damper, an inertial actuator, a mechanical structure, a transducer for the residual force, a controller, a power amplifier and a shaker. The system input, $u(t)$ is the position of the mobile part (magnet) of the inertial actuator, the output $y(t)$ is the residual force measured by a force sensor. The transfer function between the disturbance force $(\delta(t))$ and the residual force $(y(t))$ is called primary path. The plant transfer function $\left(G=q^{-d} \frac{B}{A}\right)$ between the input of the inertial actuator $(u(t))$ and the residual force is called secondary path. The parametric model of the secondary path can be straightforwardly obtained by system identification techniques. The sampling frequency is $f_{s}=800 \mathrm{~Hz}$

The frequency range of operation is between 50 and 95 $\mathrm{Hz}$. In this frequency range, 1 to 3 narrow band disturbances are introduced to the system. The objective is to strongly attenuate these disturbances. Outside the operation zone, there are robustness constraints in terms of modulus margin and noise amplification. Basically the modulus of the sensitivity functions should be kept at very low values. Specifications for the "waterbed" effect are also considered by imposing a 


\section{CONFIDENTIAL. Limited circulation. For review only.}

maximum allowed amplification. See [6] for more details of benchmark specifications and measurement procedures.

\section{A. Plant and controller description}

Consider the adaptive regulation scheme depicted in fig. 2 where the IIR YK-parametrized controller is shown. The linear case with known disturbances will be considered subsequently in order to clarify the plant and controller structure (the adaptive loop is dropped out).

The structure of the identified linear time-invariant discrete-time model of the plant (the secondary path) used for controller design is:

$$
G\left(z^{-1}\right)=\frac{z^{-d} B\left(z^{-1}\right)}{A\left(z^{-1}\right)}=\frac{z^{-d-1} B^{*}\left(z^{-1}\right)}{A\left(z^{-1}\right)},
$$

with $d$ is equal to the plant integer time delay (number of sampling periods),

$$
\begin{aligned}
A\left(z^{-1}\right) & =1+a_{1} z^{-1}+\cdots+a_{n_{A}} z^{-n_{A}} ; \\
B\left(z^{-1}\right) & =b_{1} z^{-1}+\cdots+b_{n_{B}} z^{-n_{B}}=z^{-1} B^{*}\left(z^{-1}\right) ; \\
B^{*}\left(z^{-1}\right) & =b_{1}+\cdots+b_{n_{B}} z^{-n_{B}+1},
\end{aligned}
$$

where $A\left(z^{-1}\right), B\left(z^{-1}\right), B^{*}\left(z^{-1}\right)$ are polynomials in the complex variable $z^{-1}$ and $n_{A}, n_{B}$ and $n_{B}-1$ represent their orders ${ }^{1}$. Details on system identification of the models considered in this paper can be found in [11], [9], [8].

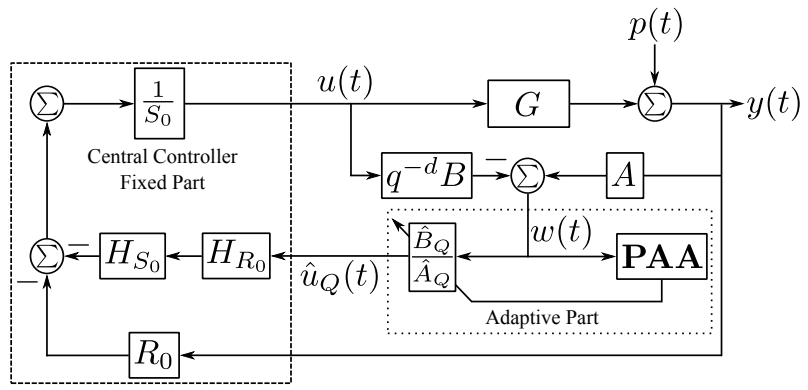

Fig. 2. Direct adaptive scheme using an IIR YK-parametrization of the controller. Dashed box: fixed part, Point-dash box: adaptive part.

The controller used in this paper corresponds to an IIR Youla-Kučera parametrized $R S$ polynomial digital controller ([7], [11] - see also figure 2). The controller is divided in a fixed (constant) part which will assign part of the closed loop poles and an IIR-YK filter $\left(B_{Q}\left(z^{-1} / B_{Q}\left(z^{-1}\right)\right.\right.$, which will compensate the effect of the disturbance by introducing the internal model of the disturbance in the controller (polynomial $B_{Q}\left(z^{-1}\right)$ ) and a polynomial $B_{Q}\left(z^{-1}\right.$ which will introduce new poles to the closed loop and will have a strong influence upon the "waterbed" effect on the shape of the sensitivity functions. When the disturbances are of unknown frequency, the parameters of the IIR-YK filter will be adapted (the estimated values will be denoted by $\hat{A}_{Q}$ and $\hat{B}_{Q}$ ).

In this scheme, the central controller is described through $R_{0}\left(z^{-1}\right)$ and $S_{0}\left(z^{-1}\right)$, which are polynomials in $z^{-1}$ having

\footnotetext{
${ }^{1}$ The complex variable $z^{-1}$ will be used for characterizing the system's behavior in the frequency domain and the delay operator $q^{-1}$ will be used for describing the system's behavior in the time domain.
}

the orders $n_{R_{0}}$ and $n_{S_{0}}$, respectively, with the following expressions:

$$
\begin{aligned}
& R_{0}=r_{0}^{0}+r_{1}^{0} z^{-1}+\ldots+r_{n_{R_{0}}}^{0} z^{-n_{R_{0}}}=R_{0}^{\prime}\left(z^{-1}\right) \cdot H_{R_{0}}\left(z^{-1}\right) ; \\
& S_{0}=1+s_{1}^{0} z^{-1}+\ldots+s_{n_{S_{0}}}^{0} z^{-n_{S_{0}}}=S_{0}^{\prime}\left(z^{-1}\right) \cdot H_{S_{0}}\left(z^{-1}\right),
\end{aligned}
$$

where $H_{R_{0}}$ and $H_{S_{0}}$ are pre-specified parts of the controller (used for example to incorporate the internal model of a disturbance or to open the loop at certain frequencies). $R_{0}\left(z^{-1}\right)$ and $S_{0}\left(z^{-1}\right)$ are minimal degree solutions of

$$
P_{0}\left(z^{-1}\right)=A\left(z^{-1}\right) S_{0}\left(z^{-1}\right)+z^{-d} B\left(z^{-1}\right) R_{0}\left(z^{-1}\right),
$$

where $P_{0}\left(z^{-1}\right)$ defines the nominal closed loop poles related to the central controller.

Under the Youla Kucera parametrization the controller polynomials are defined as follows ${ }^{2}$ :

$$
\begin{aligned}
& R=A_{Q} R_{0}+H_{S_{0}} H_{R_{0}} A B_{Q} \\
& S=A_{Q} S_{0}-H_{S_{0}} H_{R_{0}} z^{-d} B B_{Q}
\end{aligned}
$$

where the optimal $Q$-filter has the following structure:

$$
Q\left(z^{-1}\right)=\frac{B_{Q}\left(z^{-1}\right)}{A_{Q}\left(z^{-1}\right)}=\frac{b_{0}^{Q}+b_{1}^{Q} z^{-1}+\cdots+b_{n_{B} Q}^{Q} z^{-n_{B}}}{1+a_{1}^{Q} z^{1}+\cdots+a_{n_{A_{Q}}}^{Q} z^{-n_{A}}} .
$$

One defines the following sensitivity functions:

- Output sensitivity function:

$$
S_{y p}\left(z^{-1}\right)=\frac{A\left(z^{-1}\right) S\left(z^{-1}\right)}{P\left(z^{-1}\right)} ;
$$

- Input sensitivity function

$$
S_{u p}\left(z^{-1}\right)=-\frac{A\left(z^{-1}\right) R\left(z^{-1}\right)}{P\left(z^{-1}\right)},
$$

where

$$
\begin{aligned}
P & =A S+z^{-d} B R=A_{Q} P_{0} \\
& =A_{Q}\left(A S_{0}+z^{-d} B R_{0}\right)
\end{aligned}
$$

defines the poles of the closed loop (roots of $P\left(z^{-1}\right)$ ).

One can write the output of the system as:

$$
y(t)=\frac{A\left(q^{-1}\right) S\left(q^{-1}\right)}{P\left(q^{-1}\right)} \cdot p(t)=S_{y p}\left(q^{-1}\right) \cdot p(t) .
$$

\section{B. Disturbance description}

A deterministic disturbance $p(t)$ can be represented as the output of a filter excited by a Dirac pulse as

$$
p(t)=\frac{N_{p}\left(q^{-1}\right)}{D_{p}\left(q^{-1}\right)} \cdot \delta(t),
$$

where $\delta(t)$ is a Dirac pulse and $N_{p}\left(z^{-1}\right), D_{p}\left(z^{-1}\right)$ are coprime polynomials in $z^{-1}$, of degrees $n_{N_{p}}$ and $n_{D_{p}}$, respectively. In the case of persistent (stationary) disturbances the roots of $D_{p}\left(z^{-1}\right)$ are on the unit circle (which will be the case in the present context).

\footnotetext{
${ }^{2}$ The arguments $\left(z^{-1}\right)$ and $\left(q^{-1}\right)$ will be omitted in some of the following equations to make them more compact.
} 
The disturbances considered in the benchmark can in fact be represented by a sum of sinusoidal disturbances.

$$
p(t)=\sum_{i=1}^{n} C_{i} \sin \left(\omega_{i} t+\beta_{i}\right),
$$

where $\left\{C_{i}, \omega_{i}, \beta_{i}\right\} \neq 0$ and $n$ is the number of narrow band disturbances. In this case, $D_{p}\left(z^{-1}\right)$ in (15) has the expression:

$$
D_{p}\left(z^{-1}\right)=\prod_{i=1}^{n}\left(1-2 \cos \left(\omega_{i}\right) z^{-1}+z^{-2}\right),
$$

where $\omega_{i}=2 \pi f_{i} T_{s}, f_{i}$ is in $\mathrm{Hz}$ and $T_{s}=1 / f_{s}$ is the sampling time. Under this mirror structure, no matter the values of $\omega_{i}$, the roots of $D_{p}$ remains on the unit circle.

\section{INTERNAL MODEL PRINCIPLE WITH YK IIR PARAMETRIZATION}

Consider the case when the frequencies of the disturbance are known, i.e. $D_{p}\left(z^{-1}\right)$ is known and a given central controller $R_{0}\left(z^{-1}\right)$ and $S_{0}\left(z^{-1}\right)$ is already computed ${ }^{3}$.

Consider eq. (14). In order to asymptotically reject the effect of $p(t)$ over $y(t)$, the polynomial $S\left(z^{-1}\right)$ should incorporate the denominator $D_{p}\left(z^{-1}\right)$ (Internal Model Principle [4]), as is shown next:

$$
\begin{aligned}
S\left(z^{-1}\right) & =S^{\prime}\left(z^{-1}\right) \cdot H_{S}\left(z^{-1}\right) \\
& =S^{\prime}\left(z^{-1}\right) \cdot\left(H_{S_{0}}\left(z^{-1}\right) \cdot D_{p}\left(z^{-1}\right)\right) .
\end{aligned}
$$

Looking at the eq. (9), is possible to define a diophantine equation allowing to compute the optimal $Q$-IIR filter which introduces the model of the disturbance into the controller. The diophantine equation is

$$
S^{\prime} D_{p}+H_{R_{0}} z^{-d} B B_{Q}=A_{Q} S_{0}^{\prime},
$$

where the common term $H_{S_{0}}\left(z^{-1}\right)$ has been eliminated. Here $D_{p}\left(z^{-1}\right), H_{R_{0}}\left(z^{-1}\right), d, B\left(z^{-1}\right)$ and $S_{0}^{\prime}\left(z^{-1}\right)$ are known, and $B_{Q}\left(z^{-1}\right)$ and $S^{\prime}\left(z^{-1}\right)$ are unknown. In order to eq. (19) be solvable, $A_{Q}\left(z^{-1}\right)$ should be defined. Suppose temporarily that $A_{Q}\left(z^{-1}\right)$ is known and asymptotically stable (a.s.) , since this polynomial will define additional poles for the closedloop (see eq. (13)). Then, eq. (19) has a unique and minimal degree solution for $S^{\prime}\left(z^{-1}\right)$ and $B_{Q}\left(z^{-1}\right)$ with $n_{A_{Q}}+n_{S_{0}^{\prime}}-1 \leq$ $n_{D_{p}}+n_{H_{R_{0}}}+n_{B}+d-1, n_{S^{\prime}}=n_{B}+d+n_{H_{R_{0}}}-1$ and $n_{B_{Q}}=$ $n_{D_{p}}-1$.

\section{A. Structure of $A_{Q}\left(z^{-1}\right)$}

In eq. (19), the computed numerator $B_{Q}\left(z^{-1}\right)$ introduces zeros in the polynomial $S\left(z^{-1}\right)$, through the YKparametrization. This allows the rejection of the narrowband disturbance. But this does not guarantee a satisfactory modulus margin ( $\Delta M$ - see [11]) and a limited water bed effect at other frequencies.

Instead of a BSF approach used in [1], the idea is to consider a particular notch filter structure, the $\rho$ - filter

\footnotetext{
${ }^{3}$ Pole placement with sensitivity function shaping is used as computation method but any other technique can be used for the central controller. The central controller generally includes all the stable poles of the plant model, additional auxiliary real poles for robustness and a fixed part $H_{R_{0}}\left(z^{-1}\right)=$ $1-z^{-2}$ for opening the loop at $0 f_{s}$ and $0.5 f_{s}$.
}

introduced in [3]. a particular notch type structure is directly used for the YK-IIR filter:

$$
\begin{gathered}
\frac{B_{Q}\left(z^{-1}\right.}{A_{Q}\left(z^{-1}\right.}=\frac{D_{p}\left(z^{-1}\right)}{D_{p}\left(\rho z^{-1}\right)} \\
A_{Q}\left(z^{-1}\right)=D_{p}\left(\rho z^{-1}\right)=1+\rho \alpha z^{-1}+\rho^{2} z^{-2},
\end{gathered}
$$

where $\alpha=-2 \cos \left(2 \pi f T_{s}\right)$ and using a constant $\rho, 0<\rho<1$. $D_{p}\left(z^{-1}\right)$ has its roots over the unit circle (see eq. (17)). As a consequence of the change of $z^{-1}$ to $\rho z^{-1}$, the roots of $A_{Q}\left(z^{-1}\right)$ are located in the same radial line but inside of the unit circle, and therefore it is asymptotically stable. In this approach the constant $\rho$ is defined as a function of the desired attenuation. This is also a parameter for tuning the robustness, since it has influence over the waterbed effect in $S_{y p}\left(z^{-1}\right)$.

In Fig. 3 the magnitude of the frequency responses of the output sensitivity function with a single central controller but for different structures of the YK filter used for disturbance compensation are shown. The first case corresponds to the use of an YK-FIR filter (as used in [2]) for implementing the model of the disturbance and it is depicted using a dotted line. The amplifications outside of the frequency of the disturbance are important and could lead to insufficient robustness (the computed modulus margin - $\Delta M$ - is 0.0961 corresponding to an amplification of $20.6 \mathrm{~dB}$ ). The second case, represented with a dashed line, corresponds to the use of a BSF filter approach (as in [1]) for computing the optimal $B_{Q}\left(z^{-1}\right)$ and $A_{Q}\left(z^{-1}\right)$. The BSF was computed using the disturbance frequency, a desired attenuation of $-60 \mathrm{~dB}$ and a denominator damping of 0.09 (the $\Delta M$ is 0.4318 corresponding to an amplification of $7.3 \mathrm{~dB}$ ). The third case, represented with a solid line corresponds to $\rho$-notch type filter structure with $A_{Q}$ given in (21). A constant $\rho=0.97$ was used for this case (the numerator structure corresponds to the YK-FIR case considered earlier). The computed $\Delta M$ is 0.4527 corresponding to an amplification of $6.9 \mathrm{~dB}$.

Clearly the $\rho$-notch type structure requires can achieve a strong reduction of the water bed effect.Only an estimation of $\alpha$ and a given constant $\rho$ are required for directly implementing the YK-IIR filter. For that reason this type of structure has been chosen for the denominator $A_{Q}\left(z^{-1}\right)$ in order to develop an interlaced direct adaptive scheme.

\section{Parameter Adaptation Algorithms}

Consider eqs. (14) and (9). From fig. 2, the signal $w(t+1)$ is defined as follows

$$
\begin{aligned}
w(t+1) & =A\left(q^{-1}\right) y(t+1)-B^{*}\left(q^{-1}\right) u(t-d) \\
& =A\left(q^{-1}\right) p(t+1),
\end{aligned}
$$

then, the output of the closed-loop system can be expressed as follows

$$
y(t)=\frac{\left[\hat{A}_{Q} S_{0}-H_{S_{0}} H_{R_{0}} q^{-d} B \hat{B}_{Q}\right]}{\hat{A}_{Q} P_{0}} w(t) .
$$

Using the $\rho$ type YK-IIR filters it is necessary to estimate first the parameters of $D_{p}\left(z^{-1}\right)$. Then one estimates the parameters of $\hat{B}_{Q}\left(z^{-1}\right)$. 


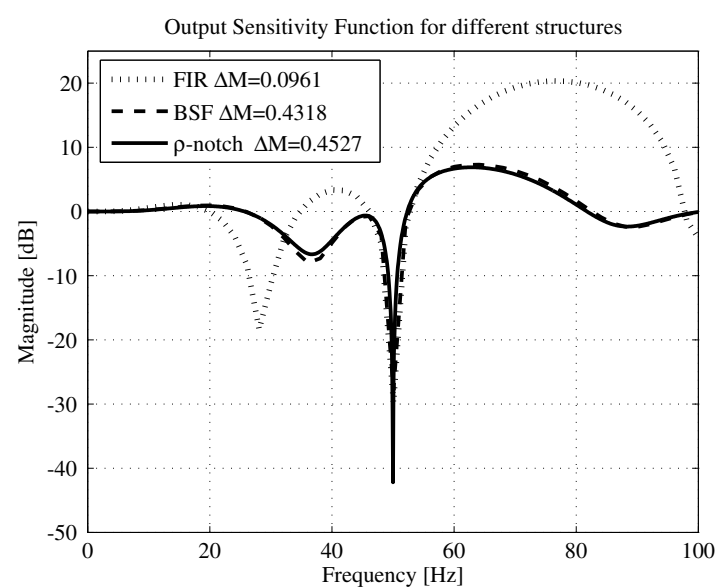

Fig. 3. Zoom of the frequency response of the output sensitivity function for different YK-filters. FIR case: dotted line, BSF case: dashed line and $\rho$-notch case: solid line.

\section{A. Estimation of $D_{p}\left(q^{-1}\right)$}

Assume that the signal $p(t)$ contains $n$ narrow-band components. $p(t)$ will then satisfy

$$
\prod_{i=1}^{n}\left(1-2 \cos \left(\omega_{i}\right) z^{-1}+z^{-2}\right) p(t)=0,
$$

where $\omega_{i}(i=1, \ldots, n)$ is the frequency of the $i^{\text {th }}$ narrow-band component in $p(t)$. Eq (24) can be equivalently written:

$$
D_{p}\left(z^{-1}\right) p(t+1)=0 .
$$

The disturbance model can be expressed by:

$$
\begin{array}{r}
p(t+1)=-\sum_{i=1}^{n-1} \alpha_{i}[p(t+1-i)+p(t+1-2 n+i)]-\cdots \\
\cdots-\alpha_{n} p(t+1-n)-p(t+1-2 n)=\theta_{D_{p}}^{T} \phi_{D_{p}}(t) .
\end{array}
$$

where the parameter vector is:

$$
\theta_{D_{p}}=\left[\alpha_{1}, \alpha_{2}, \ldots, \alpha_{n}\right]^{T} .
$$

and regressor vector at the time $t$ is:

$$
\phi_{D_{p}}(t)=\left[\phi_{1, D_{p}}(t), \phi_{2, D_{p}}(t), \phi_{n, D_{p}}(t)\right]^{T},
$$

where

$$
\begin{aligned}
& \phi_{j, D_{p}}(t)=-p(t+1-j)-p(t+1-2 n+j), j=1, \ldots, n-1 \\
& \phi_{n, D_{p}}(t)=-p(t+1-n) .
\end{aligned}
$$

Eq. (26) can then be simply represented by

$$
p(t+1)=\theta_{D_{p}}^{T} \phi_{D_{p}}(t)-p(t+1-2 n) .
$$

One defines the a priori prediction of $p(t+1)$ :

$$
\hat{p}^{0}(t+1)=\hat{\theta}_{D_{p}}^{T}(t) \phi_{D_{p}}(t)-p(t+1-2 n),
$$

where $\hat{\theta}_{D_{p}}(t)$ is the predicted parameter vector at time $t$.
The a priori prediction error is given by

$$
e^{0}(t+1)=p(t+1)-\hat{p}^{0}(t+1)=-\tilde{\theta}_{D_{p}}^{T}(t) \phi_{D_{p}}(t),
$$

where $\tilde{\theta}_{D_{p}}(t)=\hat{\theta}_{D_{p}}(t)-\theta_{D_{p}}$ is the parameter estimation error.

The following $a$ posteriori signals are defined:

- the a posteriori prediction of $p(t+1)$ :

$$
\hat{p}(t+1)=\hat{\theta}_{D_{p}}^{T}(t+1) \phi_{D_{p}}(t)-p(t+1-2 n),
$$

- the a posteriori prediction error:

$$
e(t+1)=p(t+1)-\hat{p}(t+1)=-\tilde{\theta}_{D_{p}}^{T}(t+1) \phi_{D_{p}}(t) .
$$

Equation (35) has the standard form of an a posteriori adaptation error which allows to associate the standard parameter adaptation algorithm (PAA) introduced in [7]

$$
\begin{aligned}
\hat{\theta}_{D_{p}}(t+1) & =\hat{\theta}_{D_{p}}(t)+\frac{F_{2}(t) \phi_{D_{p}}(t) e^{0}(t+1)}{1+\phi_{D_{p}}(t)^{T} F_{2}(t) \phi_{D_{p}}(t)} \\
e^{0}(t+1) & =p(t+1)-\hat{p}^{0}(t+1) \\
\hat{p}^{0}(t+1) & =\hat{\theta}_{D_{p}}^{T}(t) \phi_{D_{p}}(t)+p(t+1-2 n) \\
F_{2}(t+1)^{-1} & =\lambda_{1}(t) F_{2}(t)^{-1}-\lambda_{2}(t) \phi_{D_{p}}(t) \phi_{D_{p}}(t)^{T} \\
0<\lambda_{1}(t) & \leq 1 ; \quad 0 \leq \lambda_{2}(t)<2 ; \quad F_{2}(0)>0
\end{aligned}
$$

\section{B. Estimation of $B_{Q}\left(z^{-1}\right)$}

Consider eqs. (14) and (9). From fig. 2, the signal $w(t+1)$ is defined as follows

$$
\begin{aligned}
w(t+1) & =A\left(q^{-1}\right) y(t+1)-B^{*}\left(q^{-1}\right) u(t-d) \\
& =A\left(q^{-1}\right) p(t+1),
\end{aligned}
$$

then, the output of the closed-loop system can be expressed as follows

$$
y(t)=\frac{\left[\hat{A}_{Q} S_{0}-H_{S_{0}} H_{R_{0}} q^{-d} B \hat{B}_{Q}\right]}{\hat{A}_{Q} P_{0}} w(t) .
$$

Following the principles given in [10] and [8], it is possible to develop a direct adaptive algorithm for estimating $\hat{B}_{Q}$ provided that $\hat{A}_{Q}$ is available. Using eq. (41), the a posteriori error is defined as

$$
\begin{aligned}
\varepsilon(t+1) & =v_{1}(t+1)+\cdots \\
& \cdots\left(B_{Q}-\hat{B}_{Q}(t+1)\right) w^{f}(t+1) \cdots \\
& -\left(A_{Q}^{*}-\hat{A}_{Q}^{*}(t+1)\right) \hat{u}_{Q}^{f}(t)-A_{Q}^{*} \varepsilon(t)
\end{aligned}
$$

where

$$
\begin{aligned}
w^{f}(t+1) & =\frac{H_{S_{0}} H_{R_{0}} q^{-d} B}{P_{0}} w(t+1) \\
\hat{u}_{Q}^{f}(t) & =\frac{H_{S_{0}} H_{R_{0}} q^{-d} B}{P_{0}} \hat{u}_{Q}(t) \\
v_{1}(t+1) & =\frac{S^{\prime} H_{S_{0}} A N_{p}}{A_{Q} P_{0}} \delta(t+1)
\end{aligned}
$$

(see also Fig. 2). The signal $v_{1}(t+1)$ tends asymptotically towards zero (an asymptotically stable system excited by a Dirac pulse) and can be neglected. 
TABLE I

COMPARISON OF ALGORITHMS FOR THE ADAPTATION OF THE NUMERATOR PARAMETERS $B_{Q}\left(z^{-1}\right)$

\begin{tabular}{ccccc}
\hline $\begin{array}{c}\text { Adaptation } \\
\text { error }\end{array}$ & $\begin{array}{c}\text { Prediction } \\
\text { error }\end{array}$ & $\begin{array}{c}\text { Regressor } \\
\text { vector }\end{array}$ & $\begin{array}{c}\text { Positive } \\
\text { Real Cond. }\end{array}$ & Observations \\
$v(t+1)$ & $\varepsilon(t+1)$ & $\Phi_{1}(t)$ & $H^{\prime}\left(z^{-1}\right)$ & \\
\hline$\varepsilon(t+1)$ & Eq. (46) & $\phi_{1}(t)$ & $\frac{1}{A_{Q}}-\frac{\lambda_{2}}{2}$ & - \\
$\hat{A}_{Q} \varepsilon(t+1)$ & Eq. (46) & $\phi_{1}(t)$ & $\frac{\hat{A}_{Q}}{A_{Q}}-\frac{\lambda_{2}}{2}$ & - \\
$\varepsilon(t+1)$ & Eq. (46) & $\phi_{1}^{f}(t)$ & $\frac{\hat{A}_{Q}}{A_{Q}}-\frac{\lambda_{2}}{2}$ & - \\
$\varepsilon(t+1)$ & Eq. (46) & $\phi_{1}^{f}(t)$ & $\frac{\tilde{A}_{Q}(t)}{A_{Q}}-\frac{\lambda_{2}}{2}$ & Convergence \\
\hline
\end{tabular}

The equation for the a posteriori error takes the form

$$
\begin{aligned}
\varepsilon(t+1)= & \frac{1}{A_{Q}}\left[\theta_{1}^{T}-\hat{\theta}_{1}^{T}(t+1)\right] \phi_{1}(t+1)+\cdots \\
& \cdots+v_{1}^{f}(t+1)+v_{2}(t+1),
\end{aligned}
$$

where

$$
\begin{aligned}
v_{1}^{f}(t+1) & =\frac{1}{A_{Q}} v_{1}(t+1) \rightarrow 0, \text { since } A_{Q} \text { is a.s. } \\
v_{2}(t+1) & =\frac{1}{A_{Q}}\left(A_{Q}^{*}-\hat{A}_{Q}^{*}(t+1)\right)\left(-\hat{u}_{Q}^{f}(t)\right) \rightarrow 0, \\
\theta_{1} & =\left[b_{0}^{Q}, \cdots, b_{2 n-1}^{Q}\right]^{T} \\
\hat{\theta}_{1}(t+1) & =\left[\hat{b}_{0}^{Q}(t+1), \cdots, \hat{b}_{2 n-1}^{Q}(t+1)\right]^{T} \\
\phi_{1}(t+1) & =\left[w^{f}(t+1), \cdots, w^{f}(t+2-2 n)\right]^{T}
\end{aligned}
$$

where $n$ is the number of narrow-band disturbances. (46) has the standard form of an adaptation error equation [7], and the following PAA is proposed:

$$
\begin{aligned}
& \hat{\theta}_{1}(t+1)=\hat{\theta}_{1}(t)+F_{1}(t) \Phi_{1}(t) v(t+1) \\
& v(t+1)=\frac{\varepsilon^{0}(t+1)}{1+\Phi_{1}^{T}(t) F_{1}(t) \Phi_{1}(t)} \\
& v^{0}(t+1)=w_{1}(t+1)-\hat{\theta}_{1}^{T}(t) \Phi_{1}(t) \\
& w_{1}(t+1)=\frac{S_{0}}{P_{0}} w(t+1) \\
& F_{1}(t+1)^{-1}=\lambda_{1}(t) F_{1}(t)^{-1}-\lambda_{2}(t) \Phi_{1}(t) \Phi_{1}^{T}(t) \\
& 0<\lambda_{1}(t) \leq 1 ; 0 \leq \lambda_{2}(t)<2 ; F_{1}(0)>0
\end{aligned}
$$

Since in the equation of the a posteriori error (46 there is a term $1 / A_{Q}$, according to [7] there will be a sufficient positive real condition to be satisfied. There are several possible choices for the regressor vector $\Phi_{1}(t)$ and the filtering of the adaptation error in order to satidfy this condition. Table I gives the various options and the corresponding sufficient positive real condition. A stability analysis can be found in [?].

\section{Application to the EJC Benchmark}

The Pole Placement with sensitivity function shaping [11] is used to calculate the central controller. All the stable poles of the system are included in $P_{0}\left(z^{-1}\right)$. Also 6 real poles are added for robustness reasons. Four band stop filters have been
TABLE II

REAL-TIME RESULTS FOR THE YK-IIR ALGORIHTM - SiMPLE STEP TEST.

\begin{tabular}{cccccc}
\hline Level & Case $(\mathbf{H z})$ & $\mathbf{G A}(\mathbf{d B})$ & $\mathbf{D A}(\mathbf{d B})$ & MA $(\mathbf{d B} @ \mathbf{H z})$ & TD $\%$ \\
\hline & $\mathbf{5 0}$ & 34.5 & 40.3 & $9.3 @ 62.5$ & 92.2 \\
& $\mathbf{5 5}$ & 33.1 & 45.4 & $8.2 @ 50.0$ & 100 \\
$\mathbf{1}$ & $\mathbf{6 0}$ & 33.3 & 45.6 & $6.8 @ 125.0$ & 100 \\
$\mathbf{G A} \geq \mathbf{3 0}$ & $\mathbf{6 5}$ & 31.8 & 45.4 & $9.1 @ 56.3$ & 100 \\
$\mathbf{D A} \geq \mathbf{4 0}$ & $\mathbf{7 5}$ & 29.9 & 45.6 & $8.1 @ 131.3$ & 100 \\
$\mathbf{M A} \leq \mathbf{6}$ & $\mathbf{8 0}$ & 29.5 & 47.9 & $8.6 @ 70.3$ & 100 \\
& $\mathbf{8 5}$ & 29.5 & 43.6 & $7.7 @ 6.3$ & 100 \\
& $\mathbf{9 0}$ & 29.1 & 43.7 & $6.3 @ 117.2$ & 100 \\
& $\mathbf{9 5}$ & 27.1 & 39.0 & $7.5 @ 117.2$ & 100 \\
& $\mathbf{5 0 - 7 0}$ & 38.2 & $40.9-43.9$ & $10.3 @ 375.0$ & 100 \\
\hline $\mathbf{2}$ & $\mathbf{5 5 - 7 5}$ & 35.9 & $46.1-47.2$ & $11.9 @ 64.1$ & 100.9 \\
$\mathbf{G A} \geq \mathbf{3 0}$ & $\mathbf{6 0 - 8 0}$ & 37.8 & $45.6-45.9$ & $7.9 @ 70.3$ & 100 \\
$\mathbf{D A} \geq \mathbf{4 0}$ & $\mathbf{6 5 - 8 5}$ & 35.2 & $42.9-42.9$ & $7.9 @ 212.5$ & 100 \\
$\mathbf{M A} \leq \mathbf{7}$ & $\mathbf{7 0 - 9 0}$ & 36.1 & $43.7-44.9$ & $10.0 @ 115.6$ & 100 \\
& $\mathbf{7 5 - 9 5}$ & 35.0 & $44.9-40.0$ & $9.9 @ 128.1$ & 100 \\
\hline $\mathbf{3}$ & $\mathbf{5 0 - 6 5 - 8 0}$ & 40.1 & $38.3-39.7-43.7$ & $8.9 @ 125.0$ & 100 \\
$\mathbf{G A} \geq \mathbf{3 0}$ & $\mathbf{5 5 - 7 0 - 8 5}$ & 40.1 & $45.2-45.1-42.7$ & $7.8 @ 78.1$ & 100 \\
$\mathbf{D A} \geq \mathbf{4 0}$ & $\mathbf{6 0 - 7 5 - 9 0}$ & 38.7 & $45.2-42.2-43.3$ & $10.8 @ 78.1$ & 100 \\
$\mathbf{M A} \leq \mathbf{9}$ & $\mathbf{6 5 - 8 0 - 9 5}$ & 38.8 & $43.9-41.7-40.5$ & $10.2 @ 85.9$ & 80.9 \\
\hline
\end{tabular}

introduced in $H_{R_{0}}\left(z^{-1}\right)$ in order to shape $S_{u p}\left(z^{-1}\right)$ outside the operation zone. The loop is opened at $0 f_{s}$ and $0.5 f_{s}$. $H_{S_{0}}\left(z^{-1}\right)=1$

A value of $\rho=0.97$ has been used for all the levels and all the test. This value provides a good compromise between performance and robustness. The value is not very critical.

\section{A. Real-time results}

Table II summarizes the real-time results for the Simple Step Test. The performance objective are shown in column 1. DA is the disturbance attenuation, GA is the global attenuation, MA is the maximum amplification. TD indicates the percentage of fulfilment of the transient duration (2s). The specifications for DA,GA and TD are achieved in most of the cases. However the limits for the maximum amplification (MA) are violated for a number of cases ( But this is the case also for the schemes considered in [1], [3] and [2])

\section{B. Performance Comparison}

The results which have been presented has to be evaluated comparatively with the the most relevant schemes presented for the EJC benchmark [6]. This comparison will be done on a global basis using the procedure presented in [6]. The results presented above will be compared with those of [1], [3] and [2].

The following two global evaluation criteria are considered for comparison

- Benchmark Satisfaction Index (BSI) for steady state performance, known also as Tuning capabilities. This criterion uses the results from the Simple Step Test in order to show how "good" is the performance of a specified scheme, by measuring the fulfilment of the specifications (column Level in Table II) and assigning a percentage. 
- Complexity evaluation is done in terms of measurement of the Task Execution Time ${ }^{4}$. The value of the criterion is obtained from the average task execution time (TET) measured in the xPC-Target environment from MATLAB. Low values correspond to less complexity of the control scheme.

In Fig. 4 the comparison of the BSI for the steady state performance is presented for the four approaches mentioned. As shown, the adaptive scheme proposed in this paper (named YK-IIR) achieves the highest performance in realtime for the first level (BSI1-RT), a very good performance for the second level (BSI2-RT) and the second best (only behind [2]) for the third level (BSI3-RT).

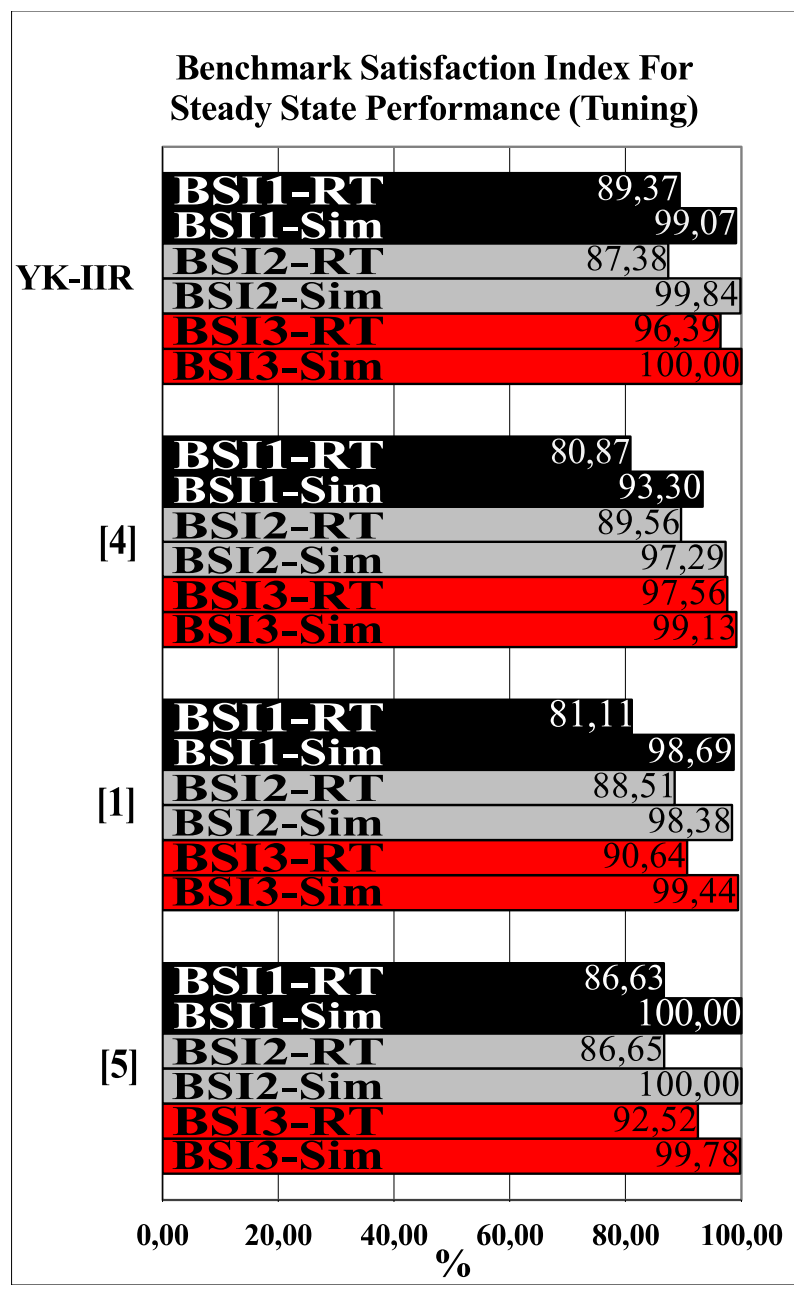

Fig. 4. Benchmark Satisfaction Index (BSI) comparison for four approaches in the three levels. $\mathrm{RT}=$ Real Time, $\mathrm{Sim}=$ Simulation.

Finally, in terms of complexity, the YK-IIR has a significant increases $\triangle$ TET compared to the one obtained in [2] (which is the lowest) using FIR Youla Kucera parametrization. However this value is still significantly smaller than the $\Delta$ TET of [1] and comparable with $\triangle$ TET of [3].

\footnotetext{
${ }^{4}$ In fact the difference between the task execution time in closed loop and in open loop is considered in the criterion.
}

\section{CONCLUDING REMARKS}

The results on this paper suggest that with an adaptive IIR Youla-Kučera Filter it is possible to achieve similar and even better results that with an FIR Youla-Kučera Filter for the strong attenuation of multiple unknown and time-varying disturbances. The advantages of using this approach is on one hand the drastic simplification of the design of the central controller and on the other hand the possibility of using a single central controller independently of the number of narrow band disturbances to be attenuated.

\section{REFERENCES}

[1] T.-B. Airimitoaie, A. Castellanos Silva, and I. D. Landau. Indirect adaptive regulation strategy for the rejection of time varying narrowband disturbances applied to a benchmark problem. European Journal of Control, 19(4), 2013.

[2] A. Castellanos Silva, I. D. Landau, and T.-B. Airimitoaie. Direct adaptive rejection of unknown time-varying narrow band disturbances applied to a benchmark problem. European Journal of Control, 19(4), 2013.

[3] X. Chen and M. Tomizuka. Adaptive model inversion for time-varying vibration rejection on an active suspension benchmark. European Journal of Control, 19(4), 2013.

[4] B.A. Francis and W.M. Wonham. The internal model principle of control theory. Automatica, 12(5):457 - 465, 1976.

[5] I. D. Landau, M. Alma, A. Constantinescu, J. J. Martinez, and M Noë. Adaptive regulation-rejection of unknown multiple narrow band disturbances (a review on algorithms and applications). Control Engineering Practice, 19(10):1168 - 1181, 2011.

[6] I. D. Landau, A. Castellanos Silva, T.-B. Airimitoaie, G. Buche, and N. Mathieu. Benchmark on adaptive regulation - rejection of unknown/time-varying multiple narrow band disturbances. European Journal of Control, 19(4), 2013.

[7] I. D. Landau, R. Lozano, M. M'Saad, and A. Karimi. Adaptive control. Springer, London, 2nd edition, 2011.

[8] I.D. Landau, M. Alma, J.J. Martinez, and G. Buche. Adaptive suppression of multiple time-varying unknown vibrations using an inertial actuator. Control Systems Technology, IEEE Transactions on, 19(6):1327 -1338, nov. 2011.

[9] I.D. Landau, A. Constantinescu, P. Loubat, D. Rey, and A. Franco. A methodology for the design of feedback active vibration control systems. Proceedings of the European Control Conference 2001, 2001. Porto, Portugal.

[10] I.D. Landau, A. Constantinescu, and D. Rey. Adaptive narrow band disturbance rejection applied to an active suspension - an internal model principle approach. Automatica, 41(4):563-574, 2005.

[11] I.D. Landau and G. Zito. Digital Control Systems - Design, Identification and Implementation. Springer, London, 2005. 